\title{
Inclusive Searches for Supersymmetry with Leptons with the ATLAS detector
}

\author{
S. Horner on behalf of the ATLAS Collaboration \\ Physikalisches Institut, \\ Albert-Ludwigs-Universität Freiburg, \\ Hermann-Herder-Straße 3, 79104 Freiburg i. Br., Germany \\ E-mail: stephan.horner@gmail.com
}

\begin{abstract}
Using the first $70 \pm 8 \mathrm{nb}^{-1}$ of integrated luminosity from $7 \mathrm{TeV}$ proton-proton collisions collected by the ATLAS detector, distributions of events containing two or more jets, missing transverse energy and at least one isolated electron or muon are analyzed in a search for new physics. No significant deviations from the Standard Model predictions are observed and good agreement with data is found indicating that the backgrounds for new physiscs are under control.
\end{abstract}

35th International Conference of High Energy Physics - ICHEP2010,

July 22-28, 2010

Paris France 


\section{Introduction}

If R-parity conserving supersymmetry (SUSY) exists at the $\mathrm{TeV}$ scale, it is expected to produce final states with jets, large missing momentum and potentially one or more isolated leptons in proton-proton collisions at LHC energies (currently $\sqrt{s}=7 \mathrm{TeV}$ ). This typical signature results from pair-produced squarks or gluinos which cascade-decay to the lightest and stable SUSY particles leaving the detector unnoticed. In the following, measurements of variables, believed to be sensitive for supersymmetry, are compared to Standard Model predictions using the first $70 \pm 8 \mathrm{nb}^{-1}$ of integrated luminosity from collisions recorded by the ATLAS detector [1], during the 2010 run. A more detailed description of the results shown below is presented in [2].

\section{Inclusive supersymmetry search with leptons}

Two separate analyses are carried out selecting events with exactly one and two or more isolated leptons respectively. Identical trigger and object selection requirements are applied on both selections, as detailed in [2]. The Standard Model backgrounds and a benchmark SUSY signal model, denoted by SU4, are studied using Monte Carlo event generation combined with a full detector simulation by GEANT4 as well as a reconstruction identical to that used for the data (for details and references see also [2]). The relevant backgrounds considered are QCD, W \& Z and $t \bar{t}$. SU4 represents a SUSY model with squark and gluino masses around $410-420 \mathrm{GeV}$ not yet excluded by the TeVatron ${ }^{1}$.

\subsection{One lepton analysis}

Only events containing at least two jets with transverse momentum $\mathrm{p}_{\mathrm{T}}>30 \mathrm{GeV}$, one isolated electron or muon with $\mathrm{p}_{\mathrm{T}}>20 \mathrm{GeV}$ and missing energy transverse to the beam $\mathrm{E}_{\mathrm{T}}^{\text {miss }}>30 \mathrm{GeV}$ are considered. Figure 1 shows the distribution of the effective mass $M_{\text {eff }}$, which is the scalar sum of the transverse momenta of both jets, the lepton and the missing $\mathrm{p}_{\mathrm{T}}$, for these events.

The QCD and W backgrounds are normalized to the number of data events in two disjunct control regions in $\mathrm{E}_{\mathrm{T}}^{\mathrm{miss}}$ and transverse mass $\mathrm{m}_{\mathrm{T}}$, the latter defined as the invariant mass of the lepton and the missing energy in the plane transverse to the beam. These regions are defined as $\mathrm{E}_{\mathrm{T}}^{\text {miss }}<40 \mathrm{GeV}$ and $\mathrm{m}_{\mathrm{T}}<40 \mathrm{GeV}$ for QCD as well as $30 \mathrm{GeV}<\mathrm{E}_{\mathrm{T}}^{\text {miss }}<50 \mathrm{GeV}$ and $40 \mathrm{GeV}<$ $\mathrm{m}_{\mathrm{T}}<80 \mathrm{GeV}$ for $\mathrm{W} . \mathrm{Z}$ and $t \bar{t}$ are normalized to their cross-sections. The yellow error band on the total Monte Carlo prediction represents the total uncertainty which results from combining the following systematic uncertainties: jet energy scale uncertainty of $7-10 \%$ depending on jet $\mathrm{p}_{\mathrm{T}}$ and $\eta, 50 \%$ uncertainty on QCD and $\mathrm{W}$ normalization, $60 \%$ on $\mathrm{Z}$ normalization, $11 \%$ luminosity uncertainty on $\mathrm{Z}$ and $t \bar{t}$ as well as the statistical uncertainty of all the Monte Carlo.

To further reduce the backgrounds from $\mathrm{QCD}$ and $\mathrm{W}$ two cuts on $\mathrm{E}_{\mathrm{T}}^{\mathrm{miss}}>30 \mathrm{GeV}$ and $\mathrm{m}_{\mathrm{T}}>$ $100 \mathrm{GeV}$ are applied resulting in the signal region distributions shown in figure 2 . Only two events remain in the electron and one event in the muon channel consistent with an expectation of $3.6 \pm 1.6$ and $2.8 \pm 1.2$ respectively.

\footnotetext{
${ }^{1}$ mSUGRA SU4: $\mathrm{m}_{0}=200 \mathrm{GeV}, \mathrm{m}_{1 / 2}=160 \mathrm{GeV}, \mathrm{A}_{0}=-400 \mathrm{GeV}, \tan \beta=10$ and $\mu>0$
} 

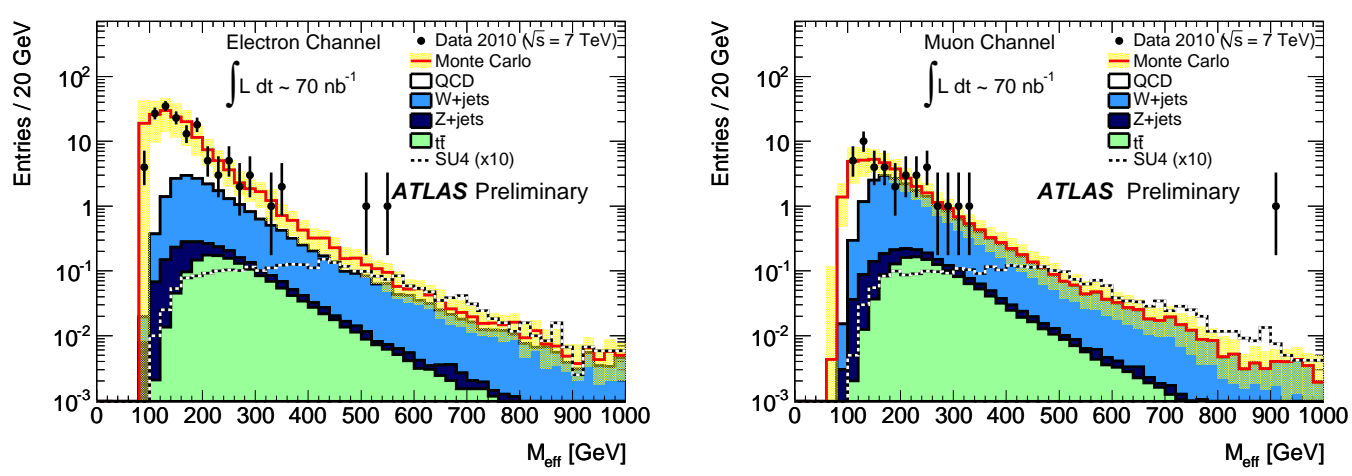

Figure 1: Effective mass distributions of events with at least two jets, an isolated lepton and missing transverse energy. The SU4 expectation is enhanced by a factor of 10 for illustrative purposes. The data are in good agreement with the Standard Model predictions.
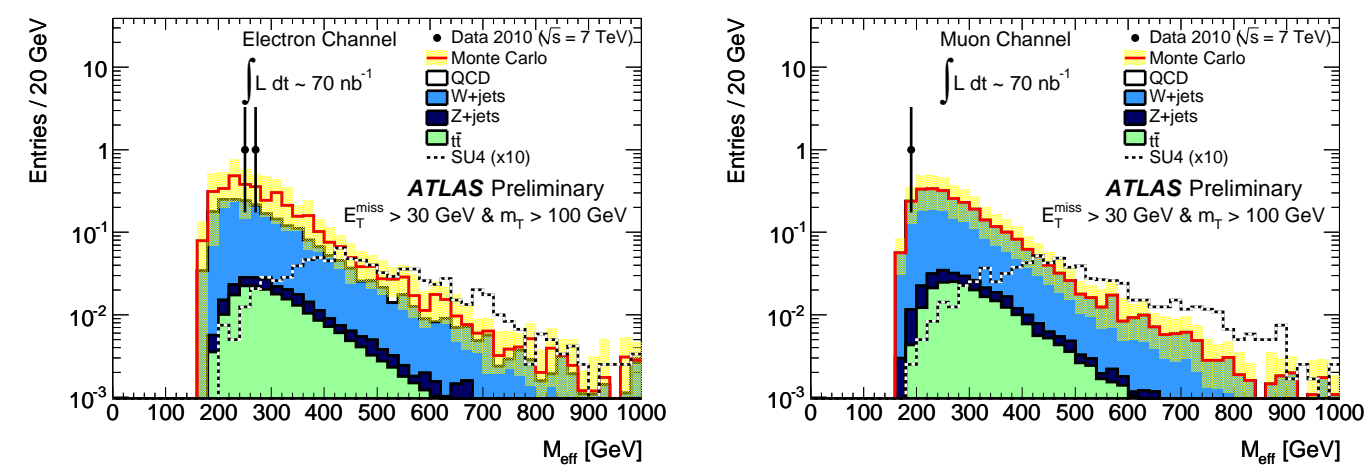

Figure 2: Effective mass distributions of events from figure 1 after requiring $\mathrm{E}_{\mathrm{T}}^{\text {miss }}$ to be bigger than $30 \mathrm{GeV}$ and $\mathrm{m}_{\mathrm{T}}$ bigger than $100 \mathrm{GeV}$ to better reject $\mathrm{QCD}$ events and leptons from $\mathrm{W}$ decays.

\subsection{Two lepton analysis}

This analysis selects events with at least two leptons having transverse momenta greater than 20 and $10 \mathrm{GeV}$ respectively. In addition, the dilepton invariant mass needs to be bigger than $5 \mathrm{GeV}$. No jet requirements are made due to the low statistics available. Figure 3 shows the missing transverse energy distributions for events containing two leptons of either opposite or same charge. Systematic uncertainties of $100 \%$ and $60 \%$ are assigned to the QCD and Z/W normalizations respectively.

The data are in agreement with the Standard Model backgrounds which have been normalized to their predicted cross-sections.

\section{Conclusion}

The first $70 \pm 8 \mathrm{nb}^{-1}$ of integrated luminosity collected by the ATLAS detector are analyzed in a search for new physics selecting events with jets, missing transverse momentum and one or two 

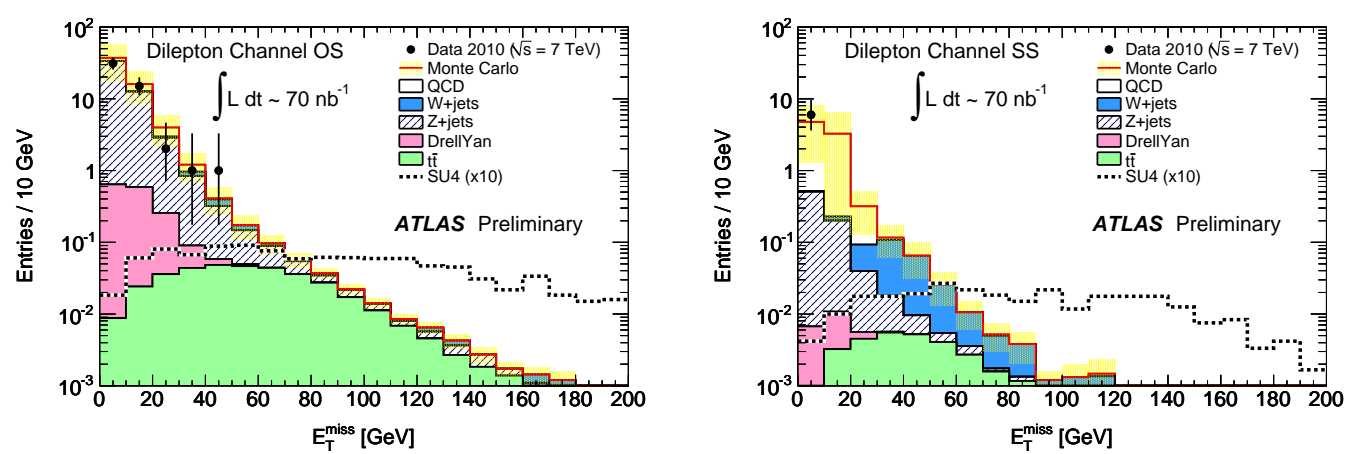

Figure 3: Missing transverse energy distributions for events containing two leptons of opposite (left plot) and same charge (right plot). The data are in agreement with the Standard Model backgrounds which have been normalized to their predicted cross-sections.

isolated leptons (electrons or muons). The data are in good agreement with the Standard Model processes and no significant deviation is observed.

\section{References}

[1] The ATLAS collaboration, G. Aad et al., JINST 3 S08003 (2008).

[2] The ATLAS collaboration, G. Aad et al., Early supersymmetry searches with jets, missing transverse momentum and one or more leptons with the ATLAS detector, [ATLAS-CONF-2010-066]. 